\title{
Design Analysis Rules to Identify Proper Noun from Bengali Sentence for Universal Networking language
}

\author{
Md. Syeful Islam \\ Computer Science and Engineering, Jahangirnagar University, Savar, Dhaka-1342, Bangladesh \\ Email: syefulislam@yahoo.com \\ Dr. Jugal Krishna Das \\ Computer Science and Engineering, Jahangirnagar University, Savar, Dhaka-1342, Bangladesh \\ Email: drdas64@yahoo.com
}

\begin{abstract}
Now-a-days hundreds of millions of people of almost all levels of education and attitudes from different country communicate with each other for different purposes and perform their jobs on internet or other communication medium using various languages. Not all people know all language; therefore it is very difficult to communicate or works on various languages. In this situation the computer scientist introduce various inter language translation program (Machine translation). UNL is such kind of inter language translation program. One of the major problem of UNL is identified a name from a sentence, which is relatively simple in English language, because such entities start with a capital letter. In Bangla we do not have concept of small or capital letters. Thus we find difficulties in understanding whether a word is a proper noun or not. Here we have proposed analysis rules to identify proper noun from a sentence and established post converter which translate the name entity from Bangla to UNL. The goal is to make possible Bangla sentence conversion to UNL and vice versa. UNL system prove that the theoretical analysis of our proposed system able to identify proper noun from Bangla sentence and produce relative Universal word for UNL.
\end{abstract}

Index Terms - Analysis window, Conditional window, Head word, Knowledge base, Morphological Analysis, Universal word.

\section{INTRODUCTION}

Today the regional economies, societies, cultures and educations are integrated through a globe-spanning network of communication and trade. This globalization trend evokes for a homogeneous platform so that each member of the platform can apprehend what other intimates and perpetuates the discussion in a mellifluous way. However the barriers of languages throughout the world are continuously obviating the whole world from congregating into a single domain of sharing knowledge and information. Therefore researcher works on various languages and tries to give a platform where multi lingual people can communicate through their native language. Researcher analyze the language structure and form structural grammar and rules which used to translate one language to other. From the very beginning the Indian linguist Panini proposed vyaakaran (a set of rules by which the language is analyzed) and gives the structure for Sanskrit language. The Astaadhyaayii of Panini (5th Century B.C) is a monumental work, comprising about four thousand short aphorisms, best known for its technical excellence. After the era of Panini various linguist works on language and proposed various technique. But the most modern theory proposed by the American linguist Noam Chomsky is universal grammar which is the base of modern language translation program. From the last few years several language-specific translation systems have been proposed. Since these systems are based on specific source and target languages, these have their own limitations. As a consequence United Nations University/Institute of Advanced Studies (UNU/IAS) were decided to develop an inter-language translation program [1]. The corollary of their continuous research leads a common form of languages known as Universal Networking Language (UNL) and introduces UNL system. UNL system is an initiative to overcome the problem of language pairs in automated translation. UNL is an artificial language that is based on Interlingua approach. UNL acts as an intermediate form computer semantic language whereby any text written in a particular language is converted to text of any other forms of languages [2].

UNL system consists of major three components: language resources, software for processing language resources (parser) and supporting tools for maintaining and operating language processing software or developing language resources. The parser of UNL system take input sentence and start parsing based on rules and convert it into corresponding universal word from word dictionary [3]. The challenge in detection of named is that such expressions are hard to analyze using UNL because they belong to the open class of expressions, i.e., there is an infinite variety and new expressions are constantly being invented. Bengali is the 
seventh popular language in the world, second in India and the national language of Bangladesh. So this is an important problem since search queries on UNL dictionary for proper nouns while all proper nouns (names) cannot be exhaustively maintained in the dictionary for automatic identification.

This work aims at attacking this problem for Bangla language, especially on the human names detection from Bengali sentence.

This research paper is organized as follows: Section II deals with the problem domain and Section III provides the theoretical analysis of The Universal Networking Language. In section IV the functioning of En-Converter is described. Section $\mathrm{V}$ provides the proposed proper noun conversion approach. Section VI gives the results corresponding to analysis. Finally Section VII draws conclusions with some remarks on future works.

\section{PROBLEM DOMAIN}

The Universal Networking Language (UNL), which is a formal language for symbolizing the sense of natural language sentences, is a specification for the exchange of information. Currently, the UNL includes 16 languages, which are the six official languages of the United Nations (Arabic, Chinese, English, French, Russian and Spanish), in addition to the ten other widely spoken languages (German, Hindi, Italian, Indonesian, Japanese, Latvian, Mongol, Portuguese, Swahili and Thai).

UNL is an electronic language for computers. It intermediates understanding among different natural languages. UNL represent sentences in the form of logical expressions, without ambiguity. These expressions are not for humans to read, but for computers. It would be hard for users to understand, and they would not need to, unless they are UNL experts. Thus, UNL is an intermediate language to be used through the internet, which allows communication among people of different languages using their mother tongue. Adding UNL to the network platforms will change the existing communication landscape. The purpose of introducing UNL in communication network is to achieve accurate exchange of information between different languages. Information has to be readable and understandable by users. Information expressed in UNL can be converted into the user's native language with higher quality and fewer mistakes than the computer translation systems. In addition, UNL, unlike natural language, is free from ambiguities.

Researchers already start works on Bengali language to include it with UNL system. Human language like Bangla is very rich in inflections, vibhakties (suffix) and karakas, and often they are ambiguous also. That is why Bangla parsing task becomes very difficult. At the same time, it is not easy to provide necessary semantic, pragmatic and world knowledge that we humans often use while we parse and understand various Bangla sentences. Bangla consists of total eighty-nine part-of-speech tags. Bangla grammatical structure generally follows the structure: subject-object-verb (S-O-V) structure. We also get useful parts of speech (POS) information from various inflections at morphological parsing. But the major problem is identifying the name from sentence and convert is very difficult. In this section we try to clear the problem domain and define some point why the processing of naming word is difficult.

In terms of native speakers, Bengali is the seventh most spoken language in the world, second in India and the national language of Bangladesh. Under the project of UNL society the Bengali linguist works on Bangla language. They already introduce some rules for UNL.

Named identification in other languages in general but Bengali in particular is difficult and challenging as:

- Unlike English and most of the European languages, Bengali lacks capitalization information.

- Bengali person names are more diverse compared to the other languages and a lot of these words can be found in the dictionary with some other specific meanings.

- Bengali is a highly inflectional language providing one of the richest and most challenging sets of linguistic and statistical features resulting in long and complex word forms.

- Bengali is a relatively free order language.

In Bengali language conversion, En-Converter parse the sentence word by word and find word from dictionary and apply rules. When En-Converter doesn't find any word from dictionary then En-Converter creates a temporary entry for this word. In the maximum case the temporary entry is name word. I give some rules to ensure that this words which is not in dictionary (temporary entry) are proper noun.

The later sections we proposed the technique of identifies the proper noun from Bangla sentence and defines a post converter for convert the Bangla name to universal word.

\section{The UnIVERSAL NeTwORKING LANGUAGE}

The Internet has emerged as the global information infrastructure, revolutionizing access to information, as well as the speed by which it is transmitted and received. With the technology of electronic mail, for example, people may communicate rapidly over long distances. Not all users, however, can use their own language for communication.

The Universal Networking Language (UNL) is an artificial language in the form of semantic network for computers to express and exchange every kind of information.

Since the advent of computers, researchers around the world have worked towards developing a system that would overcome language barriers. While lots of different systems have been developed by various organizations, each has their special representation of a given language. This results in incompatibilities between 
systems. Then, it is impossible to break language barriers in all over the world, even if we get together all the results in one system.

Against this backdrop, the concept of UNL as a common language for all computer systems was born. With the approach of UNL, the results of the past research and development can be applied to the present development, and make the infrastructure of future research and development.

The UNL consists of Universal words (UWs), Relations, Attributes, and UNL Knowledge Base. The Universal words constitute the vocabulary of the UNL, Relations and attribute constitutes the syntax of the UNL and UNL Knowledge Base constitutes the semantics of the UNL. The UNL expresses information or knowledge in the form of semantic network with hyper-node. UNL semantic network is made up of a set of binary relations, each binary relation is composed of a relation and two UWs that old the relation [4].

\section{FUNCTION OF UNL EN-CONVERTER}

To convert Bangla sentences into UNL form, we use En-Converter (EnCo), a universal converter system provided by the UNL project. The EnCo is a language independent parser, which provides a framework for morphological, syntactic and semantic analysis synchronously. Natural Language texts are analyzed sentence by sentence by using a knowledge rich lexicon and by interpreting the analysis rules. En-Converter generates UNL expressions from sentences (or lists of words of sentences) of a native language by applying Enconversion rules. In addition to the fundamental function of En-conversion, it checks the formats of rules, and outputs massages for any errors. It also outputs the information required for each stage of En-conversion in different levels. With these facilities, a rule developer can easily develop and improve rules by using En-Converter [5].

First, En-Converter converts rules from text format into binary format, or loads the binary format Enconversion rules. The rule checker works while converting rules. Once the binary format rules are made, they are stored automatically and can be used directly the next time without conversion. It is possible to choose to convert new text format rules or to use existing binary format rules.

\section{- Convert or load the rules.}

Secondly, En-Converter inputs a string or a list of morphemes / words of a sentence s native language.

\section{- Input a sentence.}

Then it starts a apply rules to the Node-list from the initial state (Fig. 1).

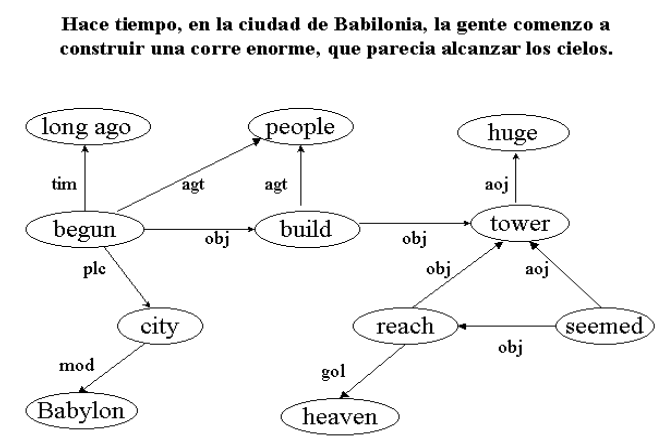

Fig.1. Sentence information is represented as a hyper-graph.

En-Converter applies En-conversion rules to the Nodelist its windows. The process of rule application is to find a suitable rule and to take actions or operate on the Nodelist in order to create a syntactic tree and UNL network using the nodes in the Analysis windows. If a string appears in the window, the system will retrieve the word dictionary and apply the rule to the candidates of word entries. If a word satisfies the conditions required for the window of a rule, this word is selected and the rule application succeeds. This process will be continued until tree and UNL network are completed and only the entry node remains in the Node-list.

- Apply the rules and retrieve the Word Dictionary.

Finally the UNL network (Node-net) is outputted to the output file in the binary relation format of UNL expression.

\section{- Output the UNL expressions.}

With the exception of the first process of rule conversion and loading, once En-Converter starts to work, it will repeat the other processes for all input sentences. It is possible to choose which and how many sentences are to be En-converted [6].

\section{A. Temporary Entries}

In the following two cases, En-Converter creates a temporary entry by assigning the attribute "TEMP"("TEMP" is the abbreviation for "Temporary").

- Except for an Arabic numeral or a blank space, if En-Converter cannot retrieve any dictionary entry from the Word Dictionary for the rest of the character string, or

- When a rule requiring the attribute "TEMP" is applied to the rest of the character string.

The temporary entry has the following format and it's UW, shown inside the double quotation "and", is assign to be the same as its headword (HW). 


$$
\text { [HW] \{\} "HW" (TEMP) <, 0, 0>; }
$$

The next section we proposed the technique of identifies the proper noun from Bangla sentence and defines a post converter for convert the Bangla name to universal word.

\section{PROPOSED PROPER NOUN CONVERSION APPROACH}

The proper noun conversion is relatively simple in English language, because such entities start with a capital letter. In Bangla we do not have concept of small or capital letters. Thus we find difficulties in understanding whether a word is a proper noun or not. For example, the Bangla word "BISWAS" can be a proper noun (i.e., a family name of a person) as well as an abstract noun (with the meaning of faith in English). For example, in order to understand the following Bangla sentence, we must need an intelligent parser. A parser takes the Bangla sentence as input and parses every sentence according to various rules [7-9].

Here we proposed a method for UNL to proper noun conversion which is a combination of dictionary-based, rule-based approaches [10]. In this approach, UNL EnConverter identifies the proper noun using rules and morphological analysis. The approaches are sequentially described here and demonstrated in Fig.2.

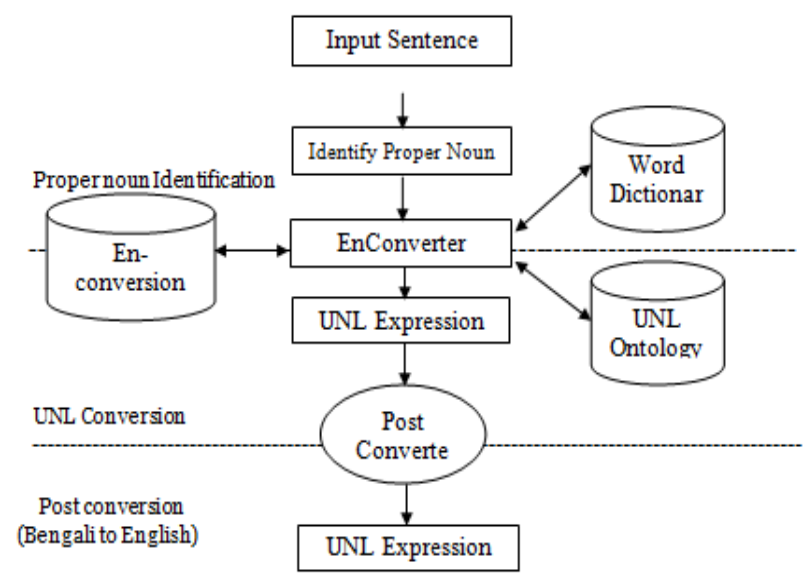

Fig.2. Flow chart of proper noun ( নাম বাচক বশিয়েয় পদ) conversion.

\section{A. Proper Noun detection approach}

In UNL system firstly take an input sentence and parse it word by word and search it from dictionary the relative word. If not found it try to recognize that the temporary word is a proper noun based on define rules. If this process is fail then morphological analysis is used. The approaches of proper noun detection are sequentially described here and demonstrated in Fig.3.

Here we describe the process in three steps.

1) Dictionary based analysis for proper noun detection

2) Rule-based analysis for proper noun detection

3) Morphological Analysis

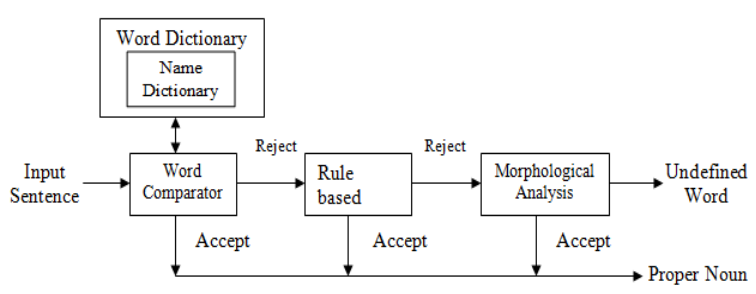

Fig.3. Proper Noun detection technique

\section{Dictionary based analysis for proper noun detection:}

If a dictionary is maintained where we try to attach most commonly used proper noun and it is known as Name dictionary. Here we describe the dictionary based proper noun detection technique sequentially.

Firstly the input sentence is processed on en-converter which finds the word on word dictionary. If the word is found then it is converted into relative universal word. If not in dictionary then En-Converter creates a temporary entry for this word.

Secondly the en-converter finds the word with flag TEMP into name dictionary. If it is found then it is conceder as the word may be noun and apply rules to ensure.

Finally if the word is not in name dictionary then it sends into morphological analyzer to conform that the word is proper noun.

\section{Rule-based analysis for proper noun detection:}

Rule-based approaches rely on some rules, one or more of which is to be satisfied by the test word. Some words which use in Bangla sentence as a part of name. Here we take a technique to identify proper noun using such word (part of name). Firstly we make dictionary entry with pof (part of name) and other relevant attribute. To identify proper noun from Bangla sentence use pof word some typical rules are given below.

Rule 1- If the parser find the following word like মোঃ, মিয়া, মিঞা, চট্টোপাধ্যায়, নুখপাধ্যায়, খান, হোসেন, হোছাইন, বহহমান, হোসাইন, ঘোষ, বোস, বসু, মিত্র, র্যায়, সরকার, খান, আহমেদ, বহমান, সটয়দ, রভোরন্ডেড, শ্রী, শ্রীযুকৃত etc. then the word is considered as the first word of name and set the status of the word first part of name (FPN). The word or collection of words after FPN with status TEMP is also considered as part of name.

Rule 2- If the parser find the following word (title words and mid-name words to human names) like চৌধুরী, মিয়া, মিঞা, চট্টোপাধ্যায়, মুখপাধ্যায়, থান, হোসেন, হোছাইন, রহমান, হোসাইন, ঘোয, বোস, বসু, মিত্র, রায়, সরকার, থান, আহমেদ, রহমান, হক etc. and কুমার, চন্দ্র, রঙন, শেথর, প্রসাদ, আলী, আলম etc. after temporary entry word. Then last part of name (LPN) and temporary entry word along with such words are likely to constitute a multi-word name(proper noun). For example, রবি বসাক, গল্লব কুমার মল্লিক are all name.

Rule 3- If there are two or more words in a sequence that represent the characters or spell like the characters of 
Bangla or English, then they belong to the name. For example, বি এ (BA), এম এ (MA), এম বি বি এস (MBBS) are all name. Note that the rule will not distinguish between a proper name and common name.

Rule 4- If a substring like বাবু, দাদা, দা, সাহেব, কাকু, গঞ, গ্রাম, পুর, গড়, নগর occurs at the end of the temporary word then temporary word is likely to be a name.

Rule 5- If a word which is in temporary entry ended with এ- র, রা, এর, র, র, রা, এরা, কে, দের, তে, য় then the word is likely to be a name.

Rule 6- If a word like- সরনী, রোড, স্ট্রিট, লেন, থানা, স্কুল, বিদ্যালয়, কলেজ, নদী, লেক, হ্রদ, সাগর, মহাসাগর, পাহাড়, পর্বত is found after temporary word then NW along with such word may belong to name. For example বিজয় সরনী, রাসেল স্ট্রেট, চিম্বুক পাহাড় all are name.

Rule 7- If the sentence containing বলেন, বললেন, বলল, শুনল, হাসল, লিথল, লিথলেন,(থলেন, দেখল after temporary word then the temporary word is likely to be a proper noun.

Rule 8- If at the end of word there are suffixes like টা,থানা, থানি, টাতে, টায়, টিকে, টাকে, টকুন, গুলা, গুলো, গুলি etc., and then word is usually not a proper noun.

\section{Morphological Analysis for proper noun detection:}

When previous two steps fail to identify proper noun or there is confusion about the word is proper noun or not then we apply morphological analysis to sure that the unknown word is proper noun. In this case we conceder the structure of words and the position of word in the sentence and identify the word type [11-12].

Rule 1- Proper noun always ended with 1st, 2nd, 5th and 6 th verbal inflexions (Bibhakti). So when parser fined an unknown word with 1st, 2nd, 5th and 6th bibhakti then the word may be proper noun [13-15].

Rule 2- From sentence structure if parser fined an unknown word is in the position of karti kaarak and word is ended with 1 st bibhakti then it is conceder as a proper noun.

Rule 3- If the unknown word position is in the position of karma kaarak and it is indirect object and word is ended with 2 nd bibhakti then it is conceder as a proper noun. But for direct object it is not a proper noun.

Rule 4- If any sentence contains more than one word ended with 1st bibhakti then the first word is with flag unknown word then must be karti kaarak and the word is proper noun.

Rule 5- In case of apaadaan kaarak, if any word in the sentence ended with 5 th bibhakti and the word is unknown then it must be noun. If most of all other's noun
Rule 6- In case of adhikaran kaarak, if any word in the sentence ended with 7th bibhakti and the word is unknown then it may be the name of place. If the word is not in dictionary then it is conceder as proper noun (name of any pace).

In that time, when En-Converter identify an word or collection of word as a proper noun and En-Converter convert into UNL expression it keep track temporary word using custom UNL relation tpl and tpr. We use two relation "tpr" and "tpl" to identify the word which should converted. The relation "tpr" use when En-Converter finds the temporary word after pof (part of name) attribute and "tpr" use when En-Converter finds the temporary word before pof (part of name ) attribute. When En-Converter found "tpr" relation then it converts the word which is after blank space. For the case of "tpl" it converts the first word. If proper noun contains only one word with attribute TEMP then using this TEMP attribute converter convert.

\section{B. Function of Post Converter}

In previous section we identify proper noun from bangle sentence and convert the sentence into corresponding intermediate UNL expression. But there is little bit problem, En-Converter convert those word which is in word dictionary. In the case of temporary word which is already identified as a proper noun or part of proper noun cannot converted and it is same as in Bengali sentence. It is difficult to other people who cannot read bangle language. So it must be converted into English for UNL expression. Post converters do this conversion. The function of post converter demonstrated in Fig. 4.

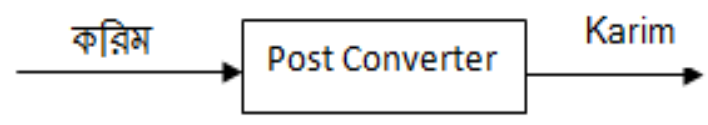

Fig.4. Function of Post Converter

\section{Proposed En-conversion process}

Here I use simple phonetic bangle to English translation method. We use same En-Converter which is used in UNL. Firstly need to create dictionary for Bengali to English conversion. Then rules are define for converter which uses these rules for conversion. When EnConverter found "tpr" relation then it converts the word which is after blank space. For the case of "tpl" it converts the first word. The functions of post converter are sequentially described here and architecture of Post converter demonstrated in Fig.5. 


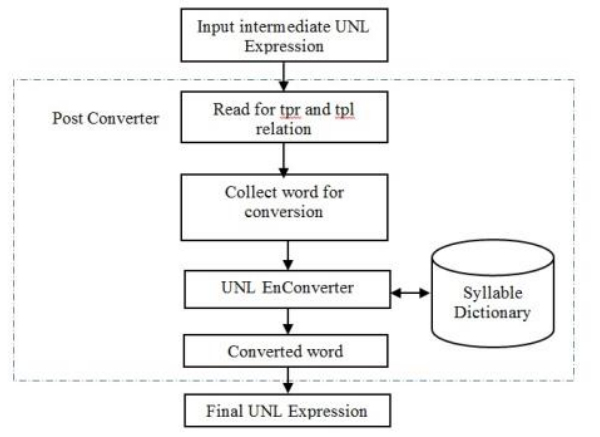

Fig.5. Architecture of Post converter (Bengali to English)

\section{Algorithm}

How post converter converts Bangla word for intermediate UNL expression to English for final UNL expression. The process are describe step by step-

Step 1: In first step the UNL expression is the inputs of post converter for find the final UNL expression.

Step 2: In second step post converter read the full expression and fined relation tpr or tpl. The relation tpr and tpl are used to identify the word which should convert.

Step 3: At third step Post converter collect Bangla word which are converted within this post converter using the help of above two relations. When EnConverter found "tpr" relation then it collects the word which is after blank space and for tpl it collects the first word.

Step 4: In this steps converter convert the word applying rules and finding the corresponding English syllable or word form syllable dictionary.

Step 5: In this step we get the converted word which is placed on final UNL expression.

Step 6: This steps is the final steps which generate final UNL expression.

Here we have listed some dictionary entries for post converter. Table 1 shows the Bengali vowel and table 2 shows the shows the Bengali consonant and the corresponding entries in dictionary. In table 3 it shows some dictionary entries for consonant plus vowel (kar). Here we only try to present how post converter converts Bengali to English. In future we define the full phonetics for Bengali to English conversion.

Table.1. Dictionary Entries for Bengali Vowel

\begin{tabular}{|c|c|}
\hline Bangla vowel & Dictionary entries \\
\hline অ & [অ] \{\} "a" (TEMP) $<, 0,0>$ \\
\hline আ & [আ] \{\} "a" (IEMP) $<, 0,0>$ \\
\hline ই & $[$ kই $]\{\}$ "i" (TEMP) $<, 0,0\rangle$ \\
\hline ঈ & [‡] \{\} "ei" (TEMP) $<, 0,0\rangle$ \\
\hline উ & [উ] \{\} "oo" (TEMP) $<, 0,0\rangle$ \\
\hline উ & [উ] \{\} "u" (TEMP) $<, 0,0>$ \\
\hline ঋ & {$[$ [ै] \{\} "rri" (TEMP) $<, 0,0>$} \\
\hline এ & [এ] \{\} "a" (TEMP) $<, 0,0>$ \\
\hline$\widehat{্}$ & {$[\widehat{\mho}]\{\}$ "oi" (TEMP) $<, 0,0>$} \\
\hline ও & {$\left[{ }^{3}\right]\{\}$ "०" (TEMP) $<, 0,0>$} \\
\hline$ঔ$ & {$[\Im]\{\}$ "ou" (TEMP) $<, 0,0>$} \\
\hline
\end{tabular}

Table.2. Dictionary Entries for Bengali Consonant

\begin{tabular}{|c|c|}
\hline Bangla consonant & Dictionary entries \\
\hline ক & [ক] \{\} "k" (TEMP) <.,0,0> \\
\hline খ & [খ] \{\} "kh" (TEMP) <.,0,0> \\
\hline গ & [গ] \{\} "g" (TEMP)<.,0,0> \\
\hline $\bar{\eta}$ & [ঘ] \{\} "gh" (TEMP) <.,0,0> \\
\hline ङ & [@] \{\} "ng" (TEMP) <.,0,0> \\
\hline $\bar{D}$ & $\left.\left[\bar{D}_{1}\right\}\right\}$ "c" (TEMP) <.,0,0> \\
\hline ছ & [ \\
\hline জ & [জ] \{\} "j" (TEMP) <.,0,0> \\
\hline ঝ & [ঝ]\{\} "jh" (TEMP) <.,0,0> \\
\hline$\Re$ & {$\left[\Re^{\prime}\right]\{\}$ "niya" (TEMP) <.,0,0> } \\
\hline ট & [ל] $[\{\}$ "t" (TEMP) <.,0,0> \\
\hline$\delta$ & [ঠ] \{\} "th" (TEMP) <.,0,0> \\
\hline ড & [Б]\{\} "d" (TEMP) <.,0,0> \\
\hline $\bar{\sigma}$ & $\left.\left[\bar{\sigma}_{]}\right\}\right\}$"dh" (TEMP) <.,0,0> \\
\hline ণ & [ণ] $\Upsilon_{\}}$"n" (TEMP) <.,0,0> \\
\hline$\overline{\mathrm{V}}$ & {$[\overline{\mathrm{V}}]\{\}$ "t" (TEMP) <.,0,0> } \\
\hline থ & [থ] \{\} "th" (TEMP) <.,0,0> \\
\hline$\overline{7}$ & {$[\overline{\bar{h}}]\{\}$ "d" (TEMP) <.,0,0> } \\
\hline $\mathcal{\psi}$ & $\left.\left[\mathcal{\Psi}_{]}\right]\right\}$"dh" (TEMP) <.,0,0> \\
\hline$\overline{7}$ & {$\left[\bar{\gamma}_{]\{\}}\right.$"n" (TEMP) <.,0,0> } \\
\hline প & {$\left[\right.$ প $_{[\}\} " \mathrm{p} "(\mathrm{TEMP})<., 0,0>}$} \\
\hline ফ & {$[\overline{\mathrm{P}}]\{\}$ "f" (TEMP) <.,0,0> } \\
\hline ব & [ব] \{\} "b" (TEMP) <.,0,0> \\
\hline ভ & [ভ] $]\{\}$ "v" (TEMP) <.,0,0> \\
\hline ম & [ম $]\{\}$ "mm" (TEMP) <.,0,0> \\
\hline য & [য]\{\} "z" (TEMP) <.,0,0> \\
\hline র & [ব্ব]\{\} "r" (TEMP) <.,0,0> \\
\hline ল & {$\left[\overline{\mathrm{m}}_{]\{\}}\right.$"1" (TEMP) <.,0,0> } \\
\hline$x$ & $\left.\left[\Upsilon_{]}\right\}\right\}$"s" (TEMP) <.,0,0> \\
\hline ষ & {$\left[ষ_{]\{\}}\right.$"sh" (TEMP) <.,0,0> } \\
\hline স & [স] \{\} "s" (TEMP) <.,0,0> \\
\hline$\overline{2}$ & {$\left[\overline{\mathcal{R}_{1}}\right]\{\}$ "h" (TEMP) <.,0,0> } \\
\hline ড় & 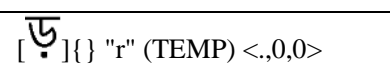 \\
\hline $\bar{G}$ & $\left.\left[\bar{\bullet}_{]}\right\}\right\}$"rh" (TEMP) <.,0,0> \\
\hline য় & [ग] $]\{\}$ "y" (TEMP) <.,0,0> \\
\hline$\oint$ & {$\left[\oint_{]\{\}}\right.$"t" (TEMP) <.,0,0> } \\
\hline
\end{tabular}


Table.3. Dictionary Entries for Bengali Consonant plus Bengali Kar

\begin{tabular}{|c|c|}
\hline $\begin{array}{c}\text { Bangla parts of } \\
\text { word }\end{array}$ & Dictionary entries \\
\hline কা & [কা] \{\} "ka" (TEMP) <.,0,0> \\
\hline কি & [কি] \{\} "ki" (TEMP) <.,0,0> \\
\hline की & [की]\{\} "kei" (TEMP) <.,0,0> \\
\hline কু & [কু] \{\} "koo" (TEMP) <.,0,0> \\
\hline কূ & [কূ] \{\} "ku" (TEMP) <.,0,0> \\
\hline কৃ & [কৃ] \{\} krriu" (TEMP) <.,0,0> \\
\hline কে & [কে] \{\} "ka" (TEMP) <.,0,0> \\
\hline কৈ & [कৈ] \{\} "koi" (TEMP) <.,0,0> \\
\hline কো & [কো] \{\} "ko" (TEMP) <.,0,0> \\
\hline কৌ & [কৌ] \{\} "kou" (TEMP) <.,0,0> \\
\hline $\bar{\infty}$ & {$[$ [ळ] \{\} "kra" (TEMP) <.,0,0> } \\
\hline क्य & [क्य] \{\} "kka" (TEMP) <.,0,0> \\
\hline
\end{tabular}

Similarly for all consonant it should need to entries in word dictionary.

\section{Example 1-}

Let's an intermediate UNL expression-

$\{$ unl $\}$

agt(read(icl>see>do,agt>person,obj>information).@entry. @present.@progress, করমি: TEMP :05)

\{/unl $\}$

Post converter firstly read the full sentence. When it finds the word "করমি" with attribute TEMP converter collect this word and push it into post converter. Then applying rules it convert into UNL word "karim". Converter parses “করমি” letter by letter.

$$
\begin{aligned}
& \text { ক : }=\text { : + অ -> Ka } \\
& \text { রা }=->\text { ri }
\end{aligned}
$$

ম $->\mathrm{m}$

That's mean “কররি" converted in "Karim”

Thus Post converter converts all proper nouns Bengali to English. Here we only try to present how post converter converts Bengali to English. In future we define the full for Bengali to English conversion.

\section{RESULT ANALYSIS}

To convert any Bangla sentence we have used the following files.

$$
\begin{array}{ll}
\checkmark & \text { Input file } \\
\checkmark & \text { Rules file } \\
\checkmark & \text { Dictionary }
\end{array}
$$

We have used an Encoder (EnCoL33.exe) and here I present some print screen of en-conversion.

Screen print shows the Encoder that produces Bangla to UNL expression or UNL to Bangla (Fig.6).

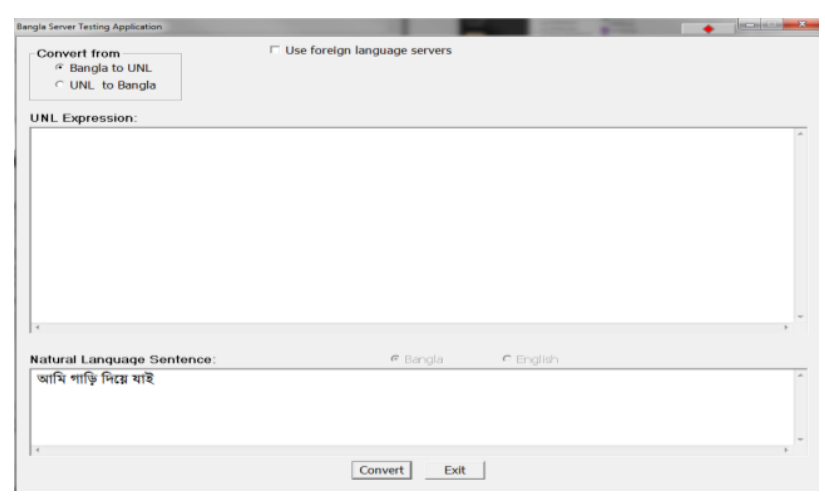

Fig.6. Encoder for En-conversion

When users click on convert button it generates corresponding UNL expression. The bellow screen shows this operation (Fig.7).

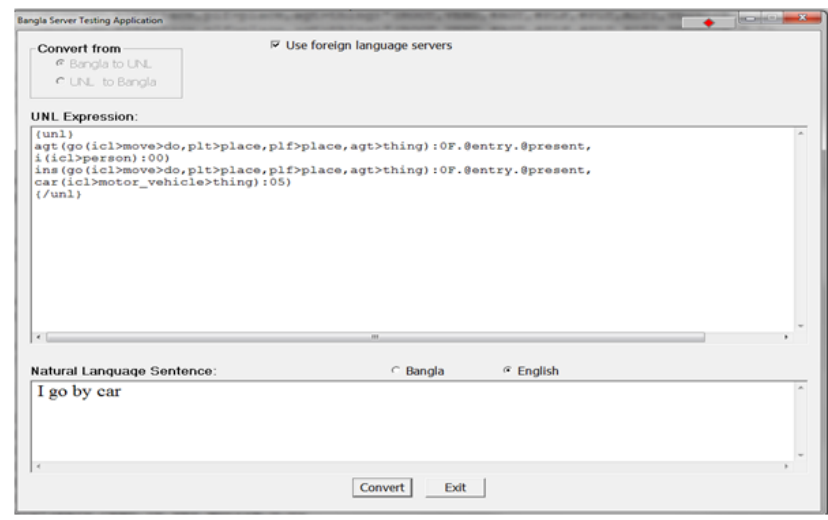

Fig.7. En-conversion

Based on the three steps pronoun detection technique we define rules for UNL system which identify the proper noun from a Bengali sentence and create relative UNL expression from the sentence.

\section{Example:}

This case is the form of a noun or pronoun used in the subject or predicate nominative. It denotes the agent of the action stated by the verb. For example, "করমি পড়তিছে" pronounce as Karim Poritechhe means "Karim is reading". Here subject Karim initiates an action. So, agent (agt) relation is made between subject "Karim" and verb "read".

The following dictionary entries are needed for conversion the sentence

- [করমি] \{\} “karim (icl>name, iof $>$ person,com $>$ male $)(\mathrm{N})$

- [9ড়"] $]\{$ "read (icl>see >do,agt>person,obj>information) (ROOT,CEND,^ALT)

- [ইতছে \{\} “’” (VI, CEND, CEG1, PRS, PRG, 3P)

; Where, $\mathrm{N}$ denotes noun, ROOT for verb root, CEND for Consonant Ended Root, ^ALT for not alternative, VI is attribute for verbal inflexion, CEG1 for Consonant Ended Group 1, PRS for present tense, PRG for progress means continuous tense and $3 \mathrm{P}$ for third person. 
But করমি is the name of a person. So it is not needed to entry on dictionary. To convert this sentence into UNL expression when En-Converter finds word which is not in dictionaries then it creates a temporary entry. So if করমি is not in dictionary then the temporary entry as like:

$$
\text { [করমি] \{\} "করমি " (TEMP) <.,0,0>; [ ] \{ \} }
$$

\section{Applied rules:}

$$
\begin{gathered}
\mathrm{R}\{:::\}\left\{\begin{array}{c}
\wedge \mathrm{PRON}, \wedge \mathrm{N}, \wedge \mathrm{VERB}, \wedge \mathrm{ROOT}, \wedge \mathrm{ADJ}, \wedge \mathrm{ADV}, \wedge \mathrm{ABY}, \wedge \\
\mathrm{KBIV}, \wedge \mathrm{BIV}:+\mathrm{N},+\mathrm{PROP}::\}(\mathrm{BLK}) \mathrm{P} 10
\end{array}\right.
\end{gathered}
$$

After applying these rules it is conceder as a proper noun. And temporary entry converted as:

\section{[করিম] \{\}$"$ করিম " (N,PROP,TEMP) <.,0,0>;.[ ] \{\}}

To convert this sentence into UNL expression morphological analysis is made between “পড়|" (por) and "ইতছে?" (itechhe) and semantic analysis is made between “করহি" (karim) and "পড়তিছে?" (poritechhe)

\section{Rule of morphological analysis:}

After applying some right shift rules when verb root " পড়|" (por) comes in the LAW and verbal inflexion " ‘তছে?" (itechhe) comes in the RAW then following rule is applied to perform morphological analysis:

$+\{$ ROOT,CEND,^ALT,^VERB:+VERB,-ROOT,+@@:: $\}$

\{VI, CEND:::\}P10;

Rule of semantic analysis:

After morphological analysis when “করমি” appears in the LAW and verb "পড়তিছে" (poritechhe) appears in the RAW the following agent (agt) relation is made between "করসি" (karim) and "পড়তিছে" (poritechhe) to complete the semantic analysis.

\{N,SUBJ::agt: \}\{VERB,\#AGT:+\&@present,+\&@progres s::\}P1; Where, $\mathrm{N}$ denotes the attributes for nouns or pronouns

The intermediate language UNL form shows that the word "করমি" same as before conversion. When the sentence "করমি পড়তিছে" converted into another language then the word "করহি" is not understandable. Because of, in UNL expression the word “করমি” is Bengali word.

$$
\begin{aligned}
& \{\text { unl }\} \\
& \text { agt(read(icl>see>do,agt>person,obj>information).@en } \\
& \text { try.@ present.@ @ progress, করমি:TEMP: 05) } \\
& \text { \{/unl }\}
\end{aligned}
$$

So this expression is invoked into Post Converter which converts this word into English using proposed simple phonetic method.

Post Converter takes the UNL expression and converts the proper noun "করমি" Bengali to English "karim".

$$
\begin{aligned}
& \text { ক }=\text { ক }+ \text { অ }>\mathrm{Ka} \\
& \text { রি }>\mathrm{ri} \\
& \text { ম }>\mathrm{m} \\
& \text { That's mean "করিম" converted in "karim" }
\end{aligned}
$$

After conversion the final UNL expression is like as-

$$
\begin{aligned}
& \{\text { unl }\} \\
& \text { agt(read(icl >see }>\text { do,agt }>\text { person,obj>information).@e } \\
& \text { ntry.@present.@progress, karim 05) } \\
& \text { \{/unl }\}
\end{aligned}
$$

Thus the UNL converter and Post converter can identify any proper noun from Bengali sentence and convert it corresponding UNL expression.

\section{CONCLUSION}

Here we have defined a procedure to identified proper noun from Bengali sentence and conversion method from bangle to UNL expression. We have also demonstrated how UNL converter identified proper noun from Bengali sentence and the UNL expression conversion by taking a sentence as an example. Here in result analysis section we demonstrate examples briefly. Here we define our work as two parts, firstly identified a proper noun from Bengali sentence and secondly convert this proper noun into UNL form. In the second parts we use a converter named as post converter which use simple phonetic method to convert Bengali to English and we only mention the simple procedure not complete. Our future plan in this regards is give the complete rules for post converter. We will also works on Bengali language and give the complete analysis rules for en-conversion program to convert Bangla to UNL language and generation rules for de-conversion program to convert any UNL documents to Bangla language.

\section{REFERENCES}

[1] http://www.undl.org last accessed on Nov 23, 2013.

[2] H. Uchida, M. Zhu, Della Senta, "A Gift for a Millennium". The United Nation University, Tokyo, Japan, 2000.

[3] H. Uchida, M. Zhu, "The Universal Networking Language (UNL) Specification Version 3.0", Technical Report, United Nations University, Tokyo, 1998.

[4] H. Uchida, M. Zhu, and T. C. D. Senta, Universal Networking Language, UNDL Foundation, International environment house, 2005/6, Geneva, Switzerland.

[5] EnConverter Specifications, version 3.3, UNL Center/ UNDL Foundation, Tokyo, Japan 2002.

[6] DeConverter Specifications, version 2.7, UNL Center/ UNDL Foundation, Tokyo, Japan 2002. 
[7] S. Abdel-Rahim, A.A. Libdeh, F. Sawalha, M.K. Odeh, "Universal Networking Language (UNL) a Means to Bridge the Digital Divide", Computer Technology Training and Indistrial Studies Center, Royal Scientific Sciety, March 2002

[8] Md. N.Y. Ali, J.K. Das, S.M. A. Al-Mamu, A.M Nurannabi, "Morphological Analysis of Bangla Words for Universal Networking Language", Third International Conference on Digital Information Management (ICDIM 2008), London, England. pp. 532-537.

[9] M.N.Y. Ali, S.A.Noor, M.H.Z. Sarker, J.K. Das, "Development of Analysis Rules for Bangla Root and Primary Suffix for Universal Networking Language".

[10] D. C. Shuniti Kumar,"Bhasha-Prakash Bangala Vyakaran", Rupa and Company Prokashoni, Calcutta, July 1999.

[11] R. T. Martins, L. H. M. Rino, M. D. G. V. Nunes, O.N. Oliveira, "The UNL distinctive features: interfaces from a NL-UNL enconverting task".

[12] Dashgupta, S., et al., "Morphological Analysis of Inflecting Compound Words in Bangla", in International Conference on Computer, and Communication Engineering (ICCIT) 2005. p. 110-117.

[13] D. S. Rameswar, "Shadharan Vasha Biggan and Bangla Vasha", Pustok Biponi Prokashoni, November 1996.

[14] H. Azad, "Bakkotottyo", Second edition, 1994, Dhaka.

[15] J. Parikh, J. Khot, S. Dave, P. Bhattacharyya, "Predicate Preserving Parsing", Department of Computer Science and Engineering , Indian Institute of Technology, Bombay.

\section{Authors' Profiles}

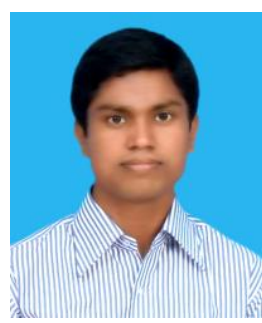

Md. Syeful Islam obtained his B.Sc. and M.Sc. in Computer Science and Engin-eering from Jahangirnagar University, Dhaka, Bangladesh in 2010 and 2011 respectively. $\mathrm{He}$ is now working as a Senior Software Engineer at Samsung R\&D Institute Bangladesh. Previously he worked as a software consultant in the Micro-Finance solutions Department of Southtech Ltd. in Dhaka, Bangladesh. His research interests are in Natural Language processing, AI, embedded computer systems and sensor networks, distributed computing and big data analysis.

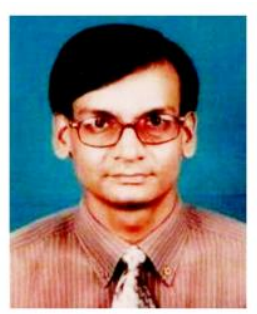

Dr. Jugal Krishna Das obtained his M.Sc. in Computer Engineering from Donetsk Technical University, Ukraine in 1989, and Ph.D. from Glushkov Institute of Cybernetics, Kiev in 1993. $\mathrm{He}$ works as a professor in the department of Computer Science and Engineering, Jahangirnagar University, Bangladesh. His research interests are in Natural Language processing, distributed computing and Computer Networking.

How to cite this paper: Md. Syeful Islam, Jugal Krishna Das,"Design Analysis Rules to Identify Proper Noun from Bengali Sentence for Universal Networking language", IJMECS, vol.6, no.8, pp.1-9, 2014.DOI: 10.5815/ijmecs.2014.08.01 